\title{
Vine Signal Extraction - an Application of Remote Sensing in Precision Viticulture
}

\author{
J.L. Smit ${ }^{*}$, G. Sithole ${ }^{1}$ and A.E. Strever ${ }^{2}$
}

(1) Geomatics Division, School of Architecture, Planning and Geomatics, University of Cape Town, Private Bag X3, Rondebosch 7701, South Africa

(2) Department of Viticulture and Oenology, Stellenbosch University, Private Bag X1, Matieland 7602, South Africa

Submitted for publication: November 2009

Accepted for publication: June 2010

Key words: Precision agriculture, precision viticulture, remote sensing, GIS, segmentation, classification

\begin{abstract}
This paper presents a study of precision agriculture in the wine industry. While precision viticulture mostly aims to maximise yields by delivering the right inputs to appropriate places on a farm in the correct doses and at the right time, the objective of this study was rather to assess vine biomass differences. The solution proposed in this paper uses aerial imagery as the primary source of data for vine analysis. The first objective to be achieved by the solution is to automatically identify vineyards blocks, vine rows, and individual vines within rows. This is made possible through a series of enhancements and hierarchical segmentations of the aerial images. The second objective is to determine the correlation of image data with the biophysical data (yield and pruning mass) of each vine. A multispectral aerial image is used to compute vegetation indices, which serve as indicators of biophysical measures. The results of the automatic detection are compared against a test field, to verify both vine location and vegetation index correlation with relevant vine parameters. The advantage of this technique is that it functions in environments where active cover crop growth between vines is evident and where variable vine canopy conditions are present within a vineyard block.
\end{abstract}

\section{INTRODUCTION}

Remote sensing has been used in the agricultural sector for many years and recent advances in imaging sensors have led to a wide array of applications using remotely sensed imagery in precision agriculture. The wine industry is one example of highvalue crop production that has benefitted from remote sensing. Precision viticulture aims to maintain maximum control over vineyard management by taking cognisance of the fact that there is variability within the vineyard (Proffit et al., 2006). It is in particular the use of multispectral imagery from airborne sensors that has seen an application in this sphere to allow for the remote determination of vineyard variability caused by differing topography, soil characteristics, management practices, plant health and meso-climates (Bramley, 2003).

The use of vegetation indices, generated from the various spectral bands of aerial multispectral images, provides a "means of capitalizing on the contrast that exists between vine biomass when measured in different wavebands" (Proffit et al., 2006). Most commonly used are the Normalized Difference Vegetation Index (NDVI) and the Plant Cell Density (PCD) or Ratio Vegetation Index (RVI). Both of these indices make use of the fact that healthy, vigorous vines will exhibit strong near-infrared reflectance and very low red reflectance (Proffit et al., 2006). These index formulations are given below in 1 and 2:

$$
\begin{aligned}
& N D V I=\left\{\frac{N I R-\operatorname{Re} d}{N I R+\operatorname{Re} d}\right\} \\
& P C D \text { or } R V I=\left\{\frac{N I R}{\operatorname{Re} d}\right\}
\end{aligned}
$$

Once the vegetation index has been calculated it is classified into a pseudo-colour index image, whereby distinct colour classes represent manageable differences in vine variability, as can be seen in Fig. 1.

The image analysis of vineyards requires, firstly, the discrimination of vineyards from neighbouring forests, homesteads and farm land; secondly, the discrimination of individual rows in the vineyard; and, lastly, the identification of individual vines in the rows. All of these operations are typically done manually. It can be seen from Fig. 1 that the pseudo-colour classification has included NDVI values for the vine rows, inter-row cover crop, shadows and bare soil. An attempt to minimise this problem can be made by modifying the classification scheme, thus effectively moving all of the pseudo-colour classes to higher NDVI values to try to isolate the NDVI values only on the vines. This thresholding process is described by Proffitt et al. (2006) and can be seen in Fig. 2, where an attempt is made to minimise the effect of inter-row cover crop, soil and shadows. It is evident in most of the vineyard blocks that obtaining a suitable threshold level is a compromise between retaining non-vine NDVI values and losing vine NDVI values. Rarely is an optimal balance achieved and hence thresholding on its own is not suitable for vine row classification.

This paper proposes a method of image segmentation to extract the vine rows from the multispectral image scene before the pseudo-colour vegetation index classification, thus reducing the classification scheme to the vine canopy only and ensuring a more reliable correlation with vine biophysical parameters such as biomass. The objective of segmentation is to automatically

*Corresponding author: e-mail: Julian.Smit@uct.ac.za [Tel.: +27 21650 3573, Fax: +27 216503572$]$ 
discriminate the vineyards from neighbouring forests and farm land, and to discriminate the rows in the vineyards, as highlighted in Fig. 3.

Once the individual rows have been discriminated, the individual vines can be identified by other means, such as analysis of the variations in the width of the rows. Once the individual vines have been identified, growth measures are estimated for them from neighbouring pixels.

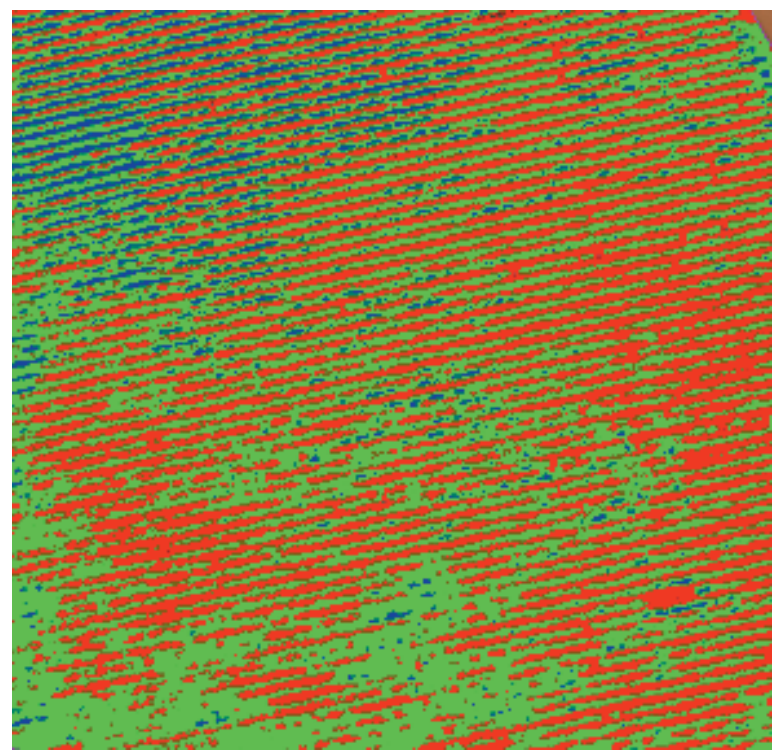

FIGURE 1

Pseudo-colour NDVI image of a portion of the vineyard test block on a Stellenbosch wine farm, where red represents low NDVI values, green represents medium NDVI values and blue represents high NDVI values.
Image segmentation can be achieved by region growing techniques, thresholding techniques, texture analysis techniques, edge detection-based techniques, graph-based techniques, or a combination of these methods. Of these, thresholding is the most efficient. There are a number of examples of segmentation applied to precision farming. An application of a thresholding segmentation technique is provided by Tellaeche et al. (2008). In their study, prior knowledge of the spectral signature of weeds was used to distinguish between weeds and crops in a field. They began with an RGB image (colour image comprised of red, green and blue bands) and converted this into a greyscale image. By studying the histogram of the greyscale image, they chose a suitable threshold. Having chosen the threshold, a binary image was created from the greyscale image, in which the weeds were shown in white and the background was shown in black. Other methods that employ thresholding are those of Rosin and Ioannidis (2003) and Tian and Slaughter (2002). To segment vineyard blocks, Delenne et al. (2006) used a Fourier analysis technique. Their method takes advantage of the periodic patterns induced by vine rows to discriminate between the vineyards and neighbouring farms and homesteads. Their method recursively seeks vineyard plots. The method is sensitive to row spacing, prone to under-segmentation if the neighbouring vineyard plots have the same row alignment, and only works on linear rows. Some other examples of methods that employ texture analysis in agriculture are provided in Wassenaar et al. (2002), Ranchin et al. (2001) and Franklin et al. (2000). Examples of an edge-based technique and region growing technique are provided by Bobillet et al. (2003) and Hall et al. (2003) respectively. A recent study to determine vineyard area Rodríguez-Pérez et al. (2008) highlights the use of supervised classification (with analyst specified "training" sites to identify vineyard components of the image) of Landsat imagery using vegetation indices, and proved to be useful at estimating vineyard areas at large scale.

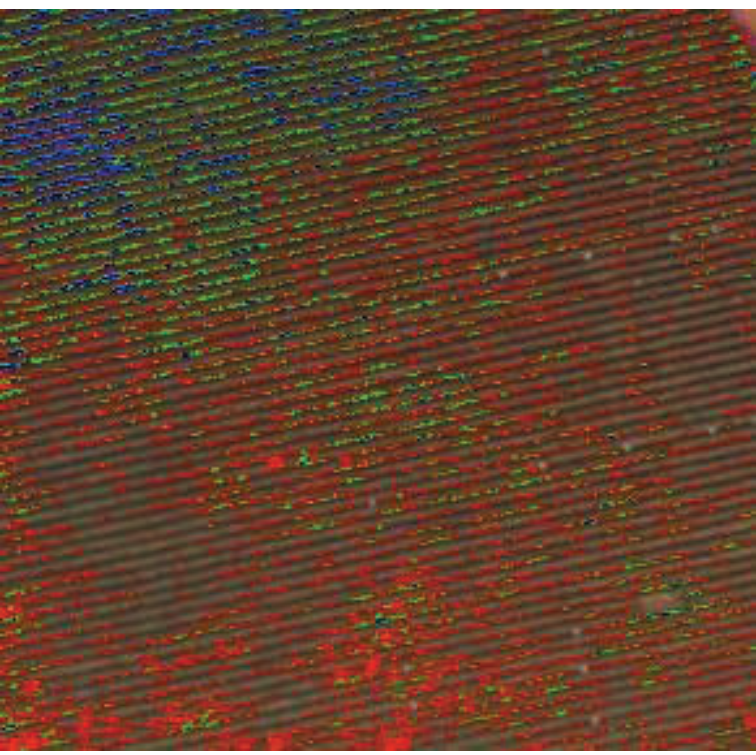

(b)

(a)

FIGURE 2

A threshold pseudo-colour NDVI image, (a) with no vine loss and (b) with vine loss as the classification scheme is modified in an attempt to remove the effects of inter-row cover crop, soil effect and shadows. 


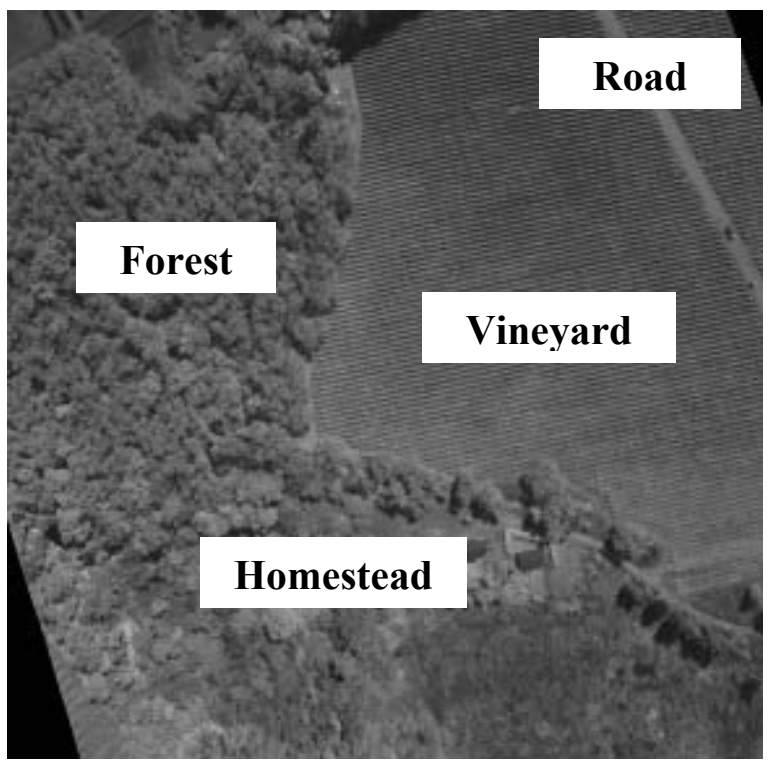

(a)

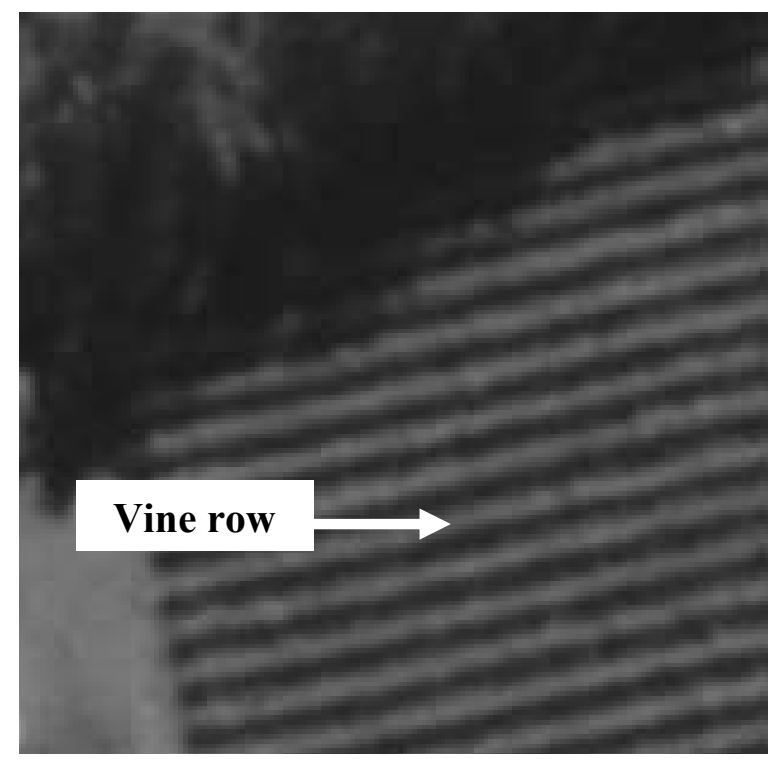

(b)

FIGURE 3

Segmentation of vineyards in an image: (a) the vineyard (the foreground) relative to forests, roads and homesteads (the background), and (b) vine rows in a vineyard.

\section{MATERIALS AND METHODS}

\section{Basic method}

The segmentation method used in this project is a combination of a thresholding and a graph-based technique. These two techniques have been chosen because they are simple, they make minimal assumptions about vine row and individual vine structures in the images, and they are adaptive. The method does not require the identification of "seed" pixels, as in region growing algorithms; it does not have to be started from a good initial state, as in active contour-based techniques; and it does not make assumptions about what defines the edge of a vine, as in edge-based techniques. The thresholding is applied to increase the contrast between the vineyard (the vine canopy) and the background (roads, "betweenrow" features, buildings, etc.). To extract the vine rows, a graph ${ }^{1}$ $\mathrm{G}(\mathrm{V}, \mathrm{E})$ is imposed on the thresholded image, in which $\mathrm{V}$ is the set of vertices in the graph and $\mathrm{E}$ is the set of edges connecting the vertices. Each vertex in the graph represents a pixel in the thresholded image, and each vertex carries the associated pixel value as an attribute. Adjacent vertices (pixels) are connected if the differences in the vertex values are below a user-defined threshold. Connecting vertices in the above fashion will yield sub-graphs in the graph G. By applying a connected components algorithm, the sub-graphs, and hence by association the segments in the thresholded image, are extracted.

\section{Discrimination of vineyards}

The vineyards are discriminated from the forest and homesteads by testing the linearity of the segments. The linearity of the segments is determined by fitting curves (low-order polynomials) to the segments and then computing the average deviation of the vertices in the segment from the computed curves. If the average deviation

\footnotetext{
1 A graph is an abstract mathematical data structure comprised of finite entities called vertices
that are connected by edges. Graphs are used in network analysis problems (Trudeau, 1993).
}

is less than the maximum expected width of a vine row, then the segment is accepted as being a vine row. This method allows the first two objectives of the segmentation to be achieved in one step, i.e. the vineyard is distinguished from the background (forest, homesteads, etc.) and the individual vine rows are extracted. Furthermore, because of the curve-fitting step, it is possible to use the method to extract curved vine rows, thus overcoming this limitation, which exists in many other techniques. This method of discrimination is more effective than a PCA (principal components analysis). Testing the linearity of segments using a PCA will require a test of the size of the second principal component. If the size of the second component is less than the maximum expected width of a vine, then a segment can be accepted as being a vine row. However, testing in this fashion will fail where the vine rows are curved, as the second component no longer represents an eigenvector perpendicular to the direction of the vine row.

\section{Source images}

The study was conducted using images of a Merlot (Vitis vinifera L. cv Merlot) clone MO 9 vineyard grafted onto R110 (Vitis Berlandieri $x$ Vitis rupestris) rootstock in the Stellenbosch grape-growing region of South Africa. The vines are planted in a $2.7 \mathrm{~m}$ (vine row spacing) x $1.5 \mathrm{~m}$ (vine spacing within a row) grid in an east-west direction and are trellised on a seven-wire movable hedge trellis system. Canopy management included shoot positioning and mechanical shoot topping. The images used in this study were collected during the 2007/2008 growing season. The test area consisted of 48 plots within the vineyard that were also used in an irrigation trial, each harbouring experimental blocks of 48 vines (12 vines in four rows).

This section discusses the image acquisition and rectification process, as well as some of the difficulties associated with segmenting the images and pre-processing the images. 


\section{Image acquisition}

Two digital SLR cameras were used for airborne image acquisition. These were a Canon 10D (Canon, 2009) with a CMOS (complementary metal oxide semiconductor) sensor, which was used to capture the colour images, and a Nikon D100 (Nikon, 2009) with a CCD (charged coupled device) sensor, which was used for the near-infrared (NIR) image capture. The Nikon D100 had been modified to capture near-infrared light (Maxmax, 2009) and used a Kodak Wratten \#89B Opaque IR filter (B\&H Photo Video, 2009) to block the visible light wavelengths. Both cameras were fitted with Sigma $14 \mathrm{~mm}$ f2/8 EX Aspherical (HSM) wideangle lenses (Photography Review, 2009) in order to optimise the camera field-of-view. The aerial photography was carried out at a height of approximately $350 \mathrm{~m}$ above the terrain, providing a nominal image ground pixel size of $20 \mathrm{~cm}$. The stereo pair of photos was taken as close to midday as possible in an attempt to minimise the effect of shadows.

\section{Image rectification}

Before the flight, both image-sensor systems were calibrated in the laboratory to determine the interior orientation parameters of the cameras (including focal length, lens distortion and principal point offset). These parameters were then used in the correction of geometric distortions in the imagery.

To obtain reliable geo-referencing of the imagery, a GPS (global positioning system) survey of the vineyard block was conducted. This included a survey of the vine row ends (to be used for verification of the segmentation), as well as the positioning of 20 photo control panels (such as can be seen in Fig. 4) in the vineyard to demarcate the test plots and serve as photo control for georeferencing.

Image geo-referencing and ortho-rectification were done using the stereo pair of multispectral images with the ERDAS Leica Photogrammetry Suite software. During this process, the colour and near-infrared images are registered band to band through the use of multiple common photo control points. The resulting colour and near-infrared ortho-rectified imagery was sampled to a ground resolution of $25 \mathrm{~cm}$.

\section{Difficulties in segmenting vineyard images}

The growth parameters for a vine are estimated from the highlighted (canopy) regions of a vine. Therefore, the assumption is made that the ideal segmentation should yield segments containing only the highlighted (vine canopy) regions of the vines.

\section{Shadows}

Sections of vines are also located in shadow areas in Fig. 5. Therefore, the segmentation also yields sections of shadowed regions. The shadow segments run parallel to the highlighted (vine

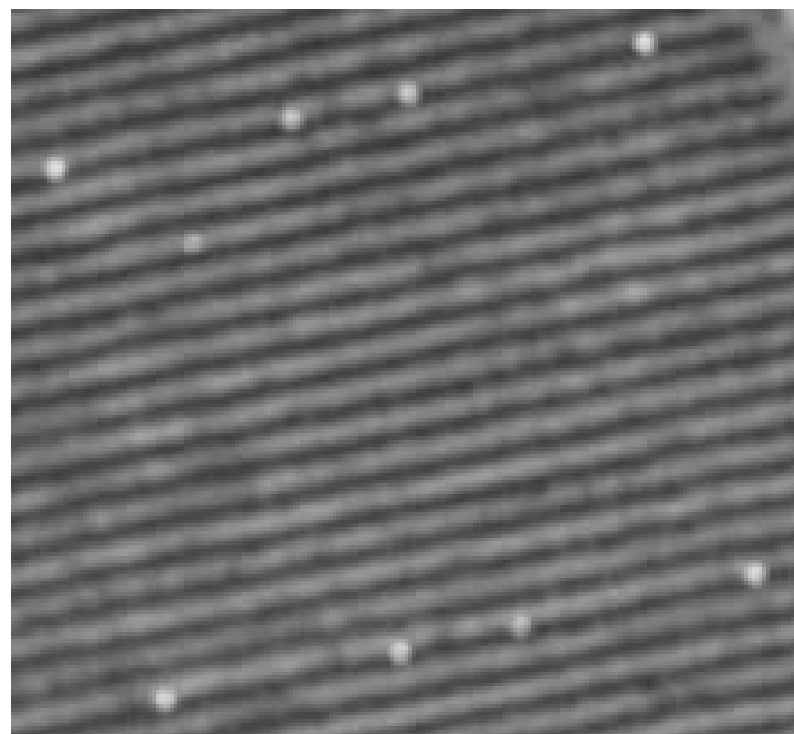

FIGURE 4

Colour image showing the white photo control panels, used for geo-referencing and test plot demarcation in a Stellenbosch wine grape vineyard.

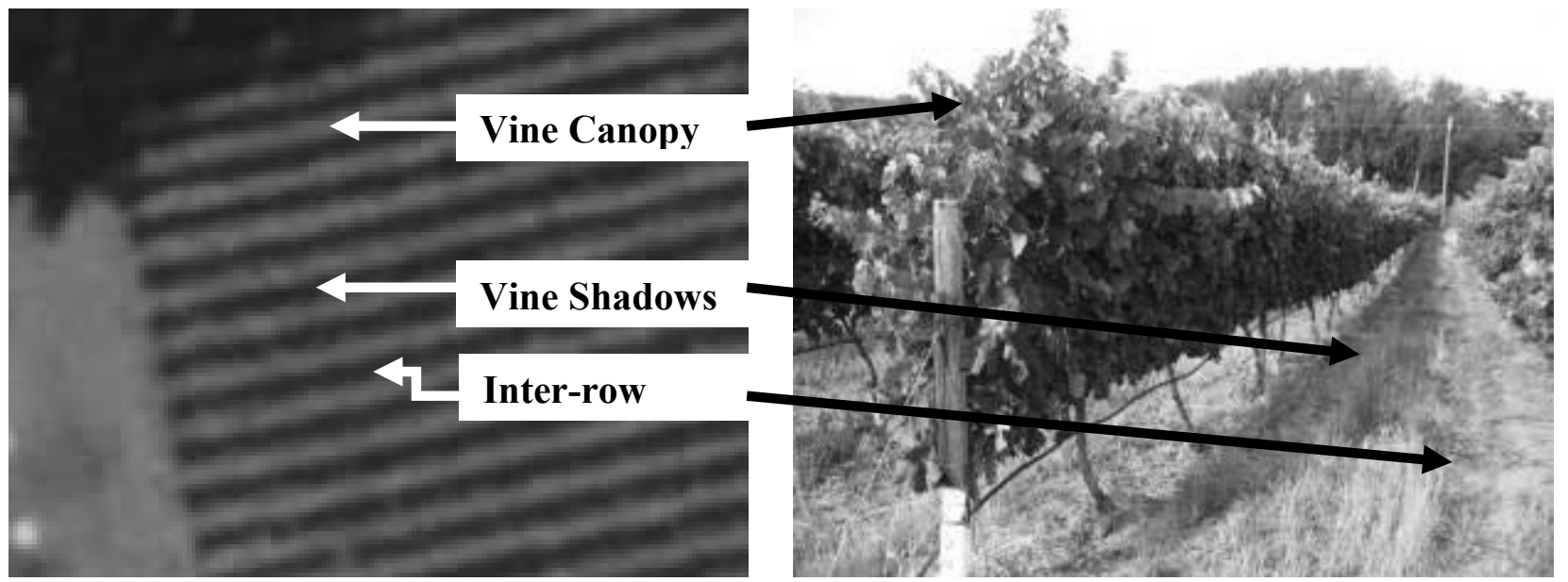

(a)

(b)

FIGURE 5

Near-infrared image showing vine highlights (canopy) versus vine shadows (a), and the situation on the ground (b). 
canopy) segments, which necessitates a post-processing step to distinguish between the highlighted (vine canopy) and shadowed segments. This discrimination is achieved by calculating the average value of the vertices in a segment. Segments with a high average value are accepted as being highlighted vine rows, and the remainder are regarded as being shadowed "vine rows".

Trees (windbreaks, etc.) also cast shadows over the vineyard, as seen in Fig. 6. The effect of this is that vineyards that should otherwise be in highlight are shadowed. This causes the vineyard area to be underestimated. The severity of this problem depends on the time and the season when the photographs were taken.

\section{Inter-rows and missing vines}

The vine rows are separated by a space measuring approximately $2.7 \mathrm{~m}$ (here called the inter-row), as shown in Fig. 7. For good discrimination between inter-rows and the highlighted vines, the inter-rows should have a spectral signature different from that of the highlighted vines (vine canopy). Depending on the season and the maintenance of the vineyard, the inter-rows may be clear, or overgrown with weeds or cover crop (annual or perennial). Overgrown inter-rows cause under-segmentation and, in the linearity test of the segments, the rows are rejected because the combined segments are wider than the expected width of one vine row segment. Inter-rows can also complicate discrimination between highlighted regions and shadowed areas. If the resolution of the image used in the segmentation is high, then segmentation should yield three parallel segments, i.e. the highlighted vine regions (vine canopy), the shaded "vine regions", and the inter-rows. Averaging the values of the vertices in these segments should result in high values, mid-values and lows for the highlights, the inter-rows and the shadows respectively. While the discrimination between segments with high and low values is fairly straightforward, the discrimination between regions with high and mid-values is problematic because of the variation in

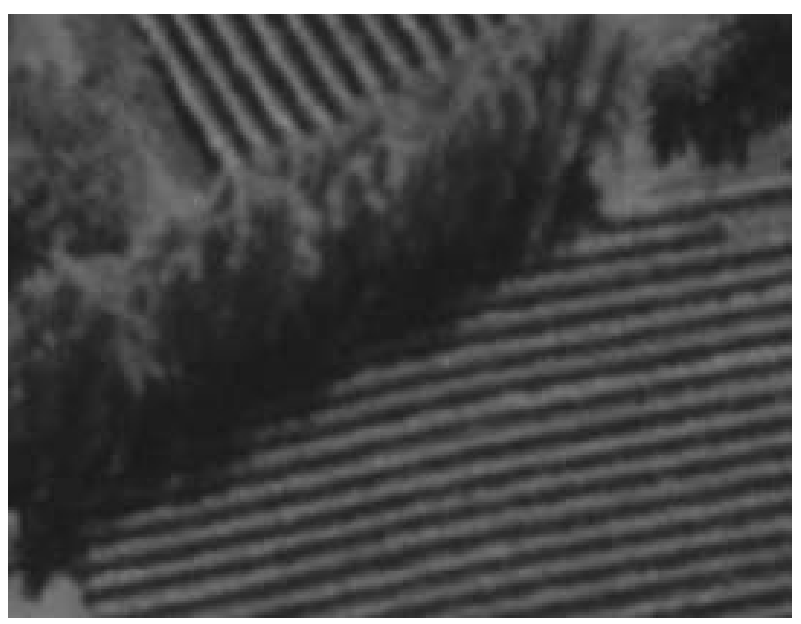

(a)

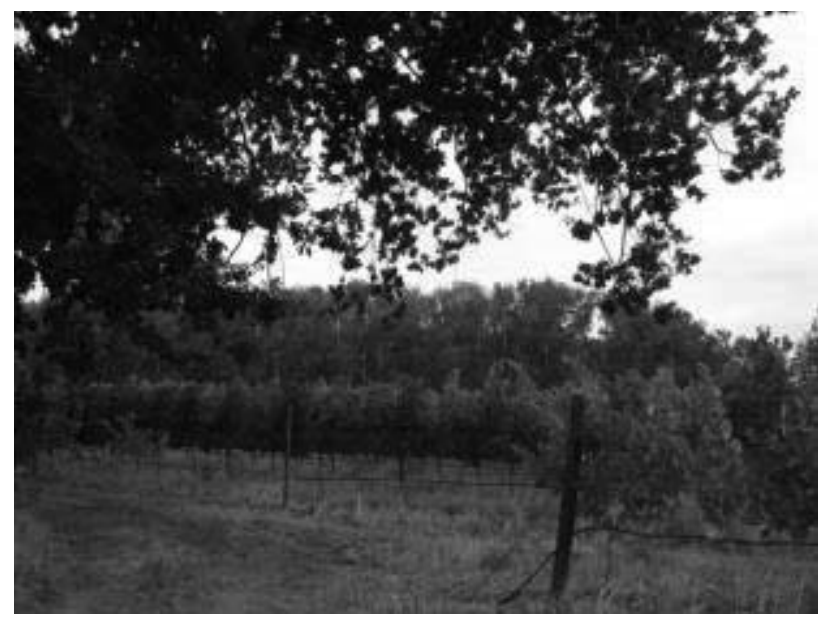

(b)

FIGURE 6

Shadows cast by trees (a), and the situation on the ground (b).

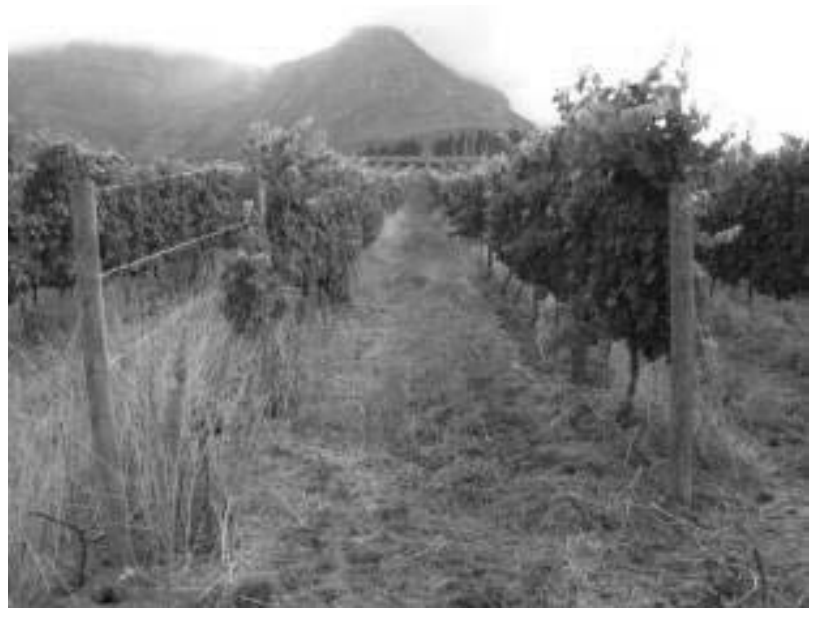

(a)

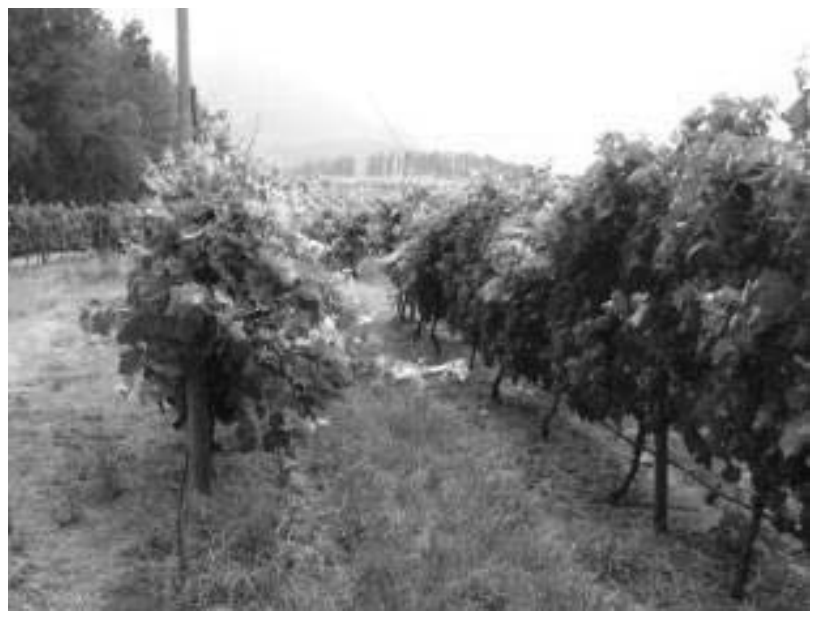

(b)

FIGURE 7

Inter-rows (the space between vine rows): A clear inter-row (a) and an overgrown inter-row (b). 


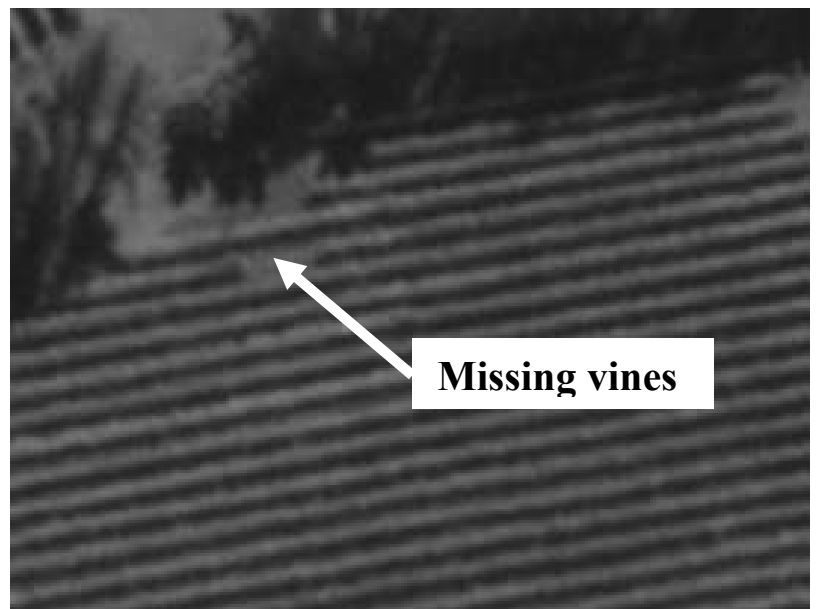

(a)

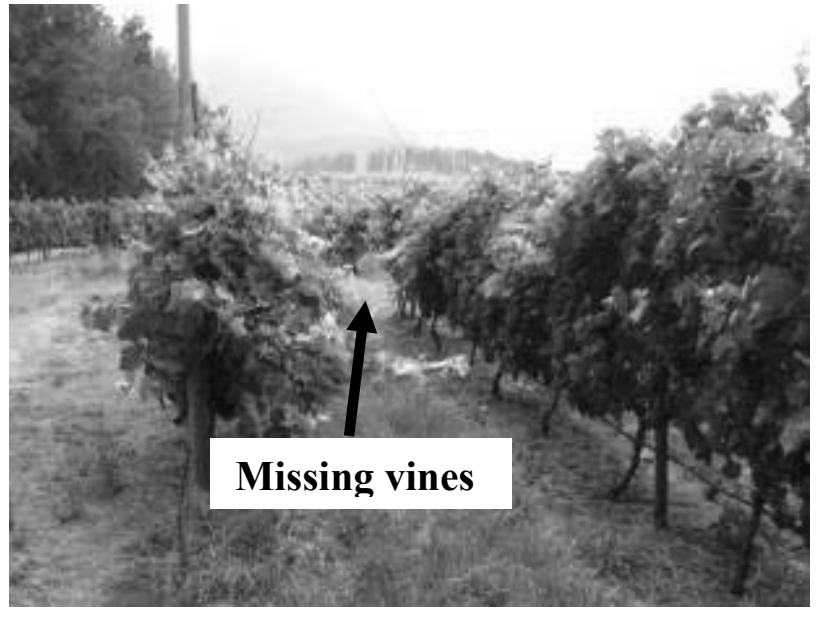

(b)

FIGURE 8

Missing vines: missing vines in the segmentation image (a), and the situation on the ground (b).

the cover crop (this can range from clear to overgrown) of the inter-rows.

A further problem associated with inter-rows is that of missing vines, as indicated in Fig. 8. Missing vines could arise, for example, from planting material problems, Eutypa wood rot problems or uprooting after infection with the leafroll virus. As can be seen in Fig. 8, missing vines can cause adjacent rows to be "combined" in the segmentation process if the inter-row is overgrown. The segment will subsequently be rejected in a linearity test to determine the linearity of the segment, because it is wider than the expected width of a vine canopy. This problem requires an additional test of segments with a high average vertex value to determine if they need to be segmented further. This essentially requires a hierarchical segmentation of the vineyard. A hierarchical segmentation was not implemented in the project reported here, but it is planned for future work.

\section{Overhanging trees}

In certain cases, trees from nearby forests or windbreaks will hang over the vines. This can cause the trees and vines to be combined into the same segment. The severity of this problem depends on whether the overhang is in shadow. The shadowed overhangs "bleed" into the shadow regions of vines and are relatively harmless. However, highlighted overhangs "bleed" into highlighted vine rows and cause adjacent vine rows to be combined into the same segment. Such highlighted overhangs necessitate a hierarchical segmentation.

\section{Pre-processing of the images}

The images used in the segmentation are not of uniform contrast. To correct this problem, local histogram equalisation must be performed on the image. Depending on the neighbourhood size, artefacts may be introduced into the image, as can be seen in Fig. 9. The likelihood of an optimal segmentation is increased when the segmentation, or discriminating parameters, are as general as possible. Increased variation, which has to be accounted for in the image scene, results in greater difficulty in the segmentation process. As indicated in Fig. 9, the vines appear most uniform in the local histogram equalisation with the 16 x 16 neighbourhood, as is to be expected. As the neighbourhood size increases, the influence of the growth variations begins to be seen. It should be noted that the source image is from the NIR (near-infrared) spectrum. Therefore, the segmentation was done on an image in which local histogram equalisation with a reasonably small neighbourhood size had been applied. The artefacts that result in the forests and in the neighbourhood of the vineyard actually aid the process of discriminating the vineyard from the neighbouring forests and homesteads. The artefacts yield many small or twisted segments that are later easily rejected with simple tests.

\section{RESULTS AND DISCUSSION}

In this section the results of the segmentation and the determination of growth measures are discussed.

\section{Segmentation}

After pre-processing the source image, the segmentation procedure as described above was applied. The threshold for the difference between adjacent vertices in the graph was set to a nominal value of five. This threshold gave good results for this particular data. More work has to be done in future permutations of the software for this threshold to be selected automatically. The vine rows segmented well, yielding long segments (Fig. 10) that can easily be discriminated from the short segments found in the forest areas. The exception to this is the roads, which also yielded long segments. However, a simple test of parallelism should be enough to distinguish roads from vine rows

The most critical step of the segmentation proved to be the preprocessing and thresholding of the source images (for this project an NIR image). Good pre-processing of the images should yield images in which the global variation between vines is minimal. In this respect, the local histogram equalisation was successful. Future work will focus on using RGB images, which are potentially better for discriminating overgrown inter-rows. 


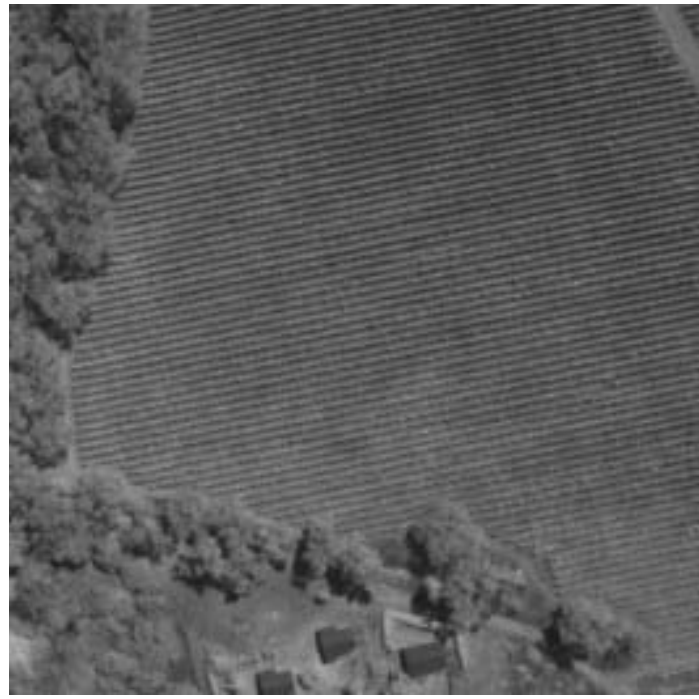

(a)

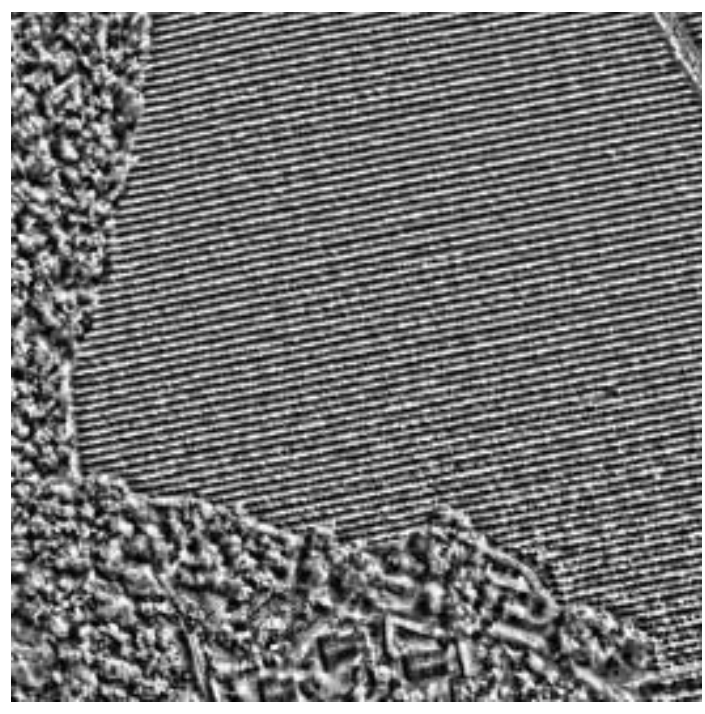

(c)

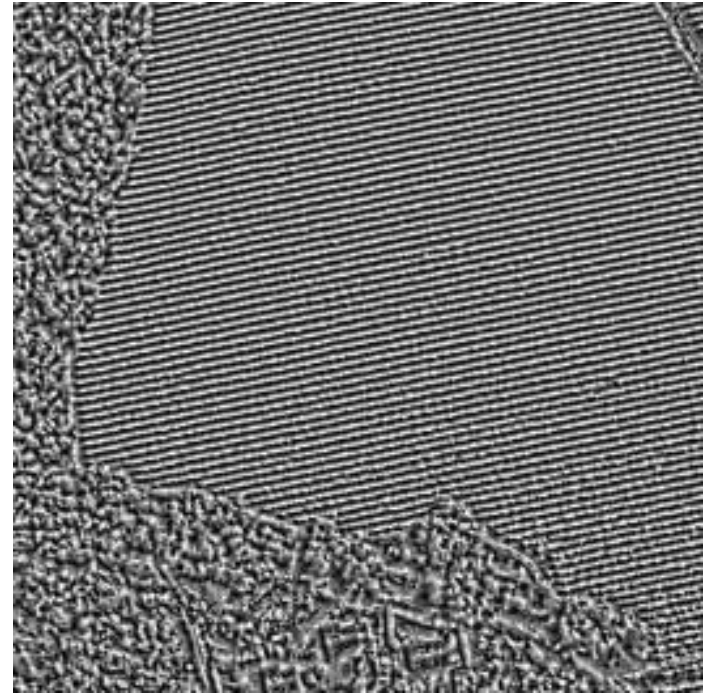

(b)

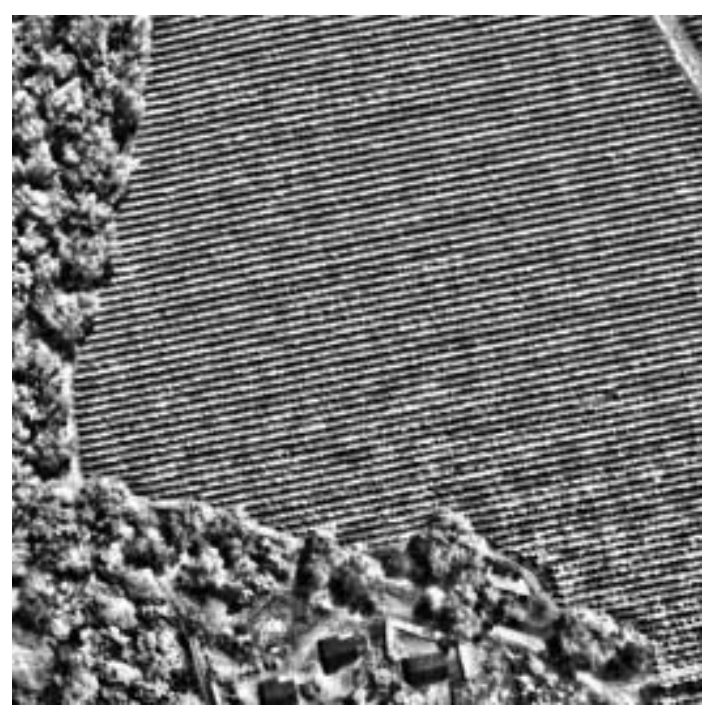

(d)

FIGURE 9

Local histogram equalisation of the source image. The original image (a). Local histogram equalisation with a $16 \mathrm{x} 16$ neighbourhood; the vine rows appear to be of uniform texture (b). Local histogram equalisation with a 32 x 32 neighbourhood; the vine rows appear to be fairly uniform, but a small influence of the global growth variation can be seen (c). Local histogram equalisation with a 64 x 64 neighbourhood; the vine rows no longer appear uniform and the influence of global growth variations can be seen clearly (d).

\section{Vine biophysical parameters}

The goal of this study was to determine measures from the radiometry of the images that can be used to estimate vine biophysical parameters. The role of the segmentation was to identify the rows of vines, and individual vines if possible. With the rows of vines identified, the next step was to identify the individual vines and obtain physical measurements for them. For the purpose of this study, a control set of vines was measured in a vineyard. The locations of these vines were determined using GPS. Furthermore, the pruning mass of the vines was determined in the winter as a measure of the seasonal above-ground biomass of the vines. Using their GPS locations, the vines were identified in the images and image-based indices were computed for the vines. Below is an explanation of how this was done.

\section{Fitting curves to the row segments}

Once the vine rows were extracted from the vineyard, the next step was to obtain the centreline of each row. The solution adopted here was to fit a low-order polynomial (second order) to each row segment. The Levenberg Marquette algorithm was used for the optimisation of the fit. Therefore, each vine row is represented by a polynomial function. The result is shown in Fig. 10(c), with the GPS vine locations superimposed. 


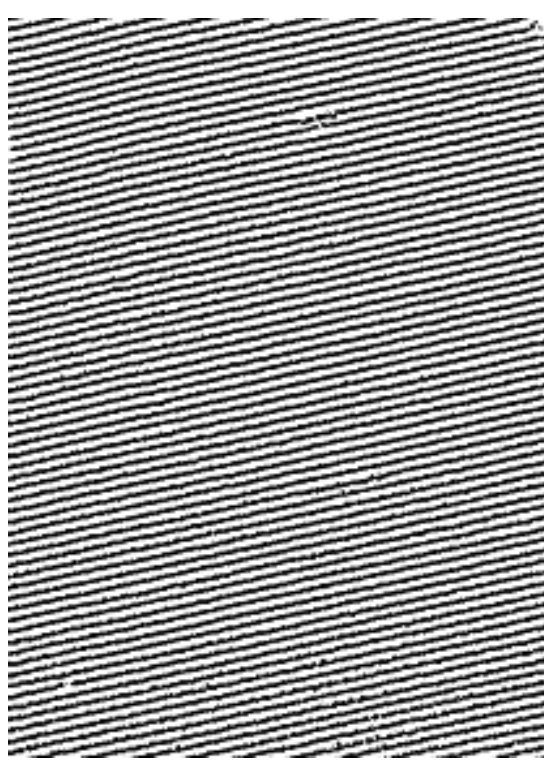

(a)

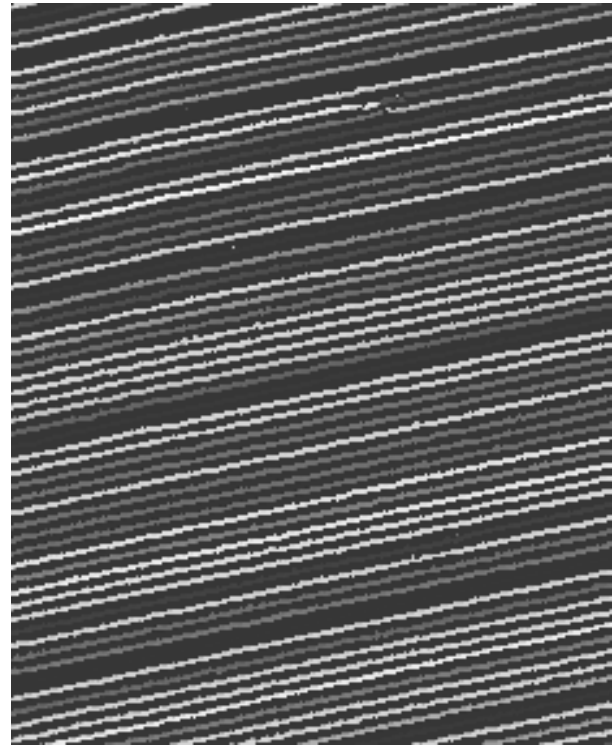

(b)

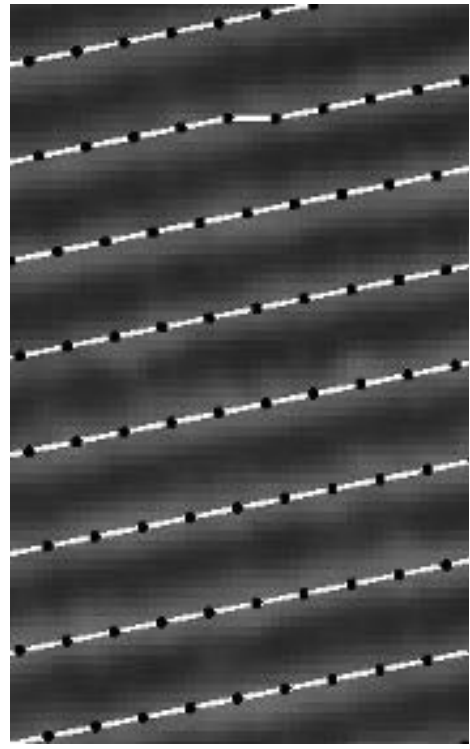

(c)

FIGURE 10

Segmentation example. The thresholded, pre-processed image (a). The segmented vine rows extracted from the pre-processed image (b). The vine row centre lines with GPS vine locations superimposed (c).

\section{Detecting and rejecting non-vine segments}

Using GPS positions, each vine was located in the image used in the segmentation. If a vine fell within a vine row segment, then all pixels in the neighbourhood of the vine, in the NIR image, were sought. The neighbourhood is defined by the radius, $r$, where $r$ is half the distance between adjacent vines in a row. Each pixel found was tested to determine if it was in the same row segment as the vine. Those pixels that were not in the same row segment were rejected. The same procedure was repeated with the RGB image.

\section{Gathering growth values for the vines}

From the pixels in the neighbourhood of a vine that cover the vine canopy (in this test case a $3 \times 3$ pixel neighbourhood was used), the following values were calculated: NDVI, PVI, mean red, mean green, mean blue, mean NIR, the mean distance to the vine and the mean distance to the vine row. The above process was repeated for every vine. This is shown in Fig. 10(c) with the GPS vine locations superimposed. The results were tabulated together with the pruning mass measurement for each vine. In Johnson et al. (2003), mention is made of the fact that vine leaf area is related to many parameters (fruit ripening rate, vine water status, fruit composition and wine quality), but also that it is not easy for grape producers to measure it, as direct measurement is time consuming and destructive. As in many other studies, these authors also indicated the good relationship between pruning mass (measured post-season in winter) and leaf area. For this reason, the measurement of pruning mass can be considered a more practical and relevant measure of vine biomass, which has been used in several remote sensing studies (Jonhnson et al., 1996; Lobitz et al., 1997; Strever, 2003).
As in Johnson et al. (2003), it was found that the sensitivity of the NDVI reaction to pruning mass on a plot level (a plot consisting of four rows of 12 vines grouped together within the vineyard) varied considerably, as is shown in Fig. 11(a), with significant improvement in the correspondence between pruning mass and NDVI per plot, shown in Fig. 11(b), when the plots were manually selected from three distinct vigour levels according to pruning mass values, seen in Fig. 12(a). In general, plot NDVI and pruning mass correlations (data not shown) were weak $\left(\mathrm{R}^{2}=0.25\right)$, but improved significantly $\left(\mathrm{R}^{2}=0.76\right)$ when plots of three distinct varying vigour levels were sub-selected from the 48 plots, as shown in Fig. 11(b) and Fig. 12(a). This may be due solely to the grouping of the selected vigour levels, as in Fig. 12(b) (this is comparable to many studies where correlations are made from sub-grouped vigour levels investigated within vineyards).

The results of the yield to NDVI correlations were similar. Correlations between row NDVI levels and the pruning mass per row were highly variable, however, with most plots not showing any correlations with NDVI values. In addition, single-vine correlations were not significant in all the cases evaluated using this segmentation method. The poor correlations found with this method are probably the result of the inability of the method to incorporate the spatial and spectral features of a vine; i.e. when a vine is smaller, the segmentation and subsequent pixel evaluation accounted for the NDVI index values of the pixels surrounding the vine, but, with the pixels limited to the segmented area, did not include a parameter to account for the number of pixels segmented. This "vine size" parameter is deemed to be just as important as the spectral value of the pixels included in the segmentation, and will be included in further studies. 


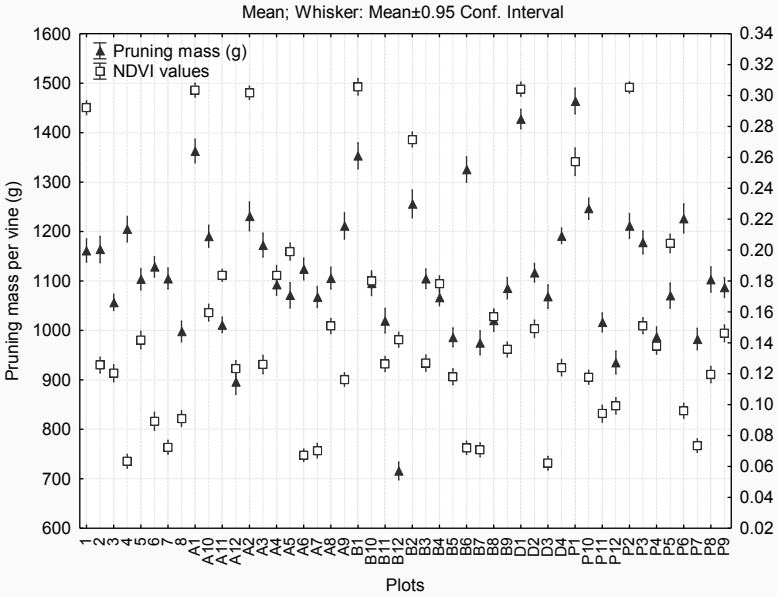

(a)

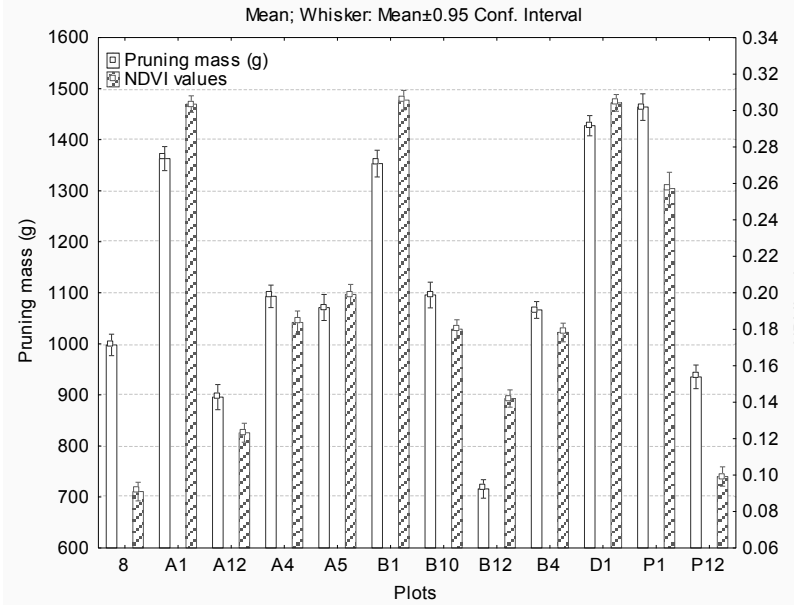

(b)

FIGURE 11

Means with error plot of pruning mass (g) per vine and NDVI values measured for the plots studied in the vineyard. Each plot consists of 48 vines (a). Means with error plot of pruning mass $(\mathrm{g})$ per vine and NDVI values measured for a sub-selection of plots in three distinct vigour levels. Each plot consists of 48 vines (b).

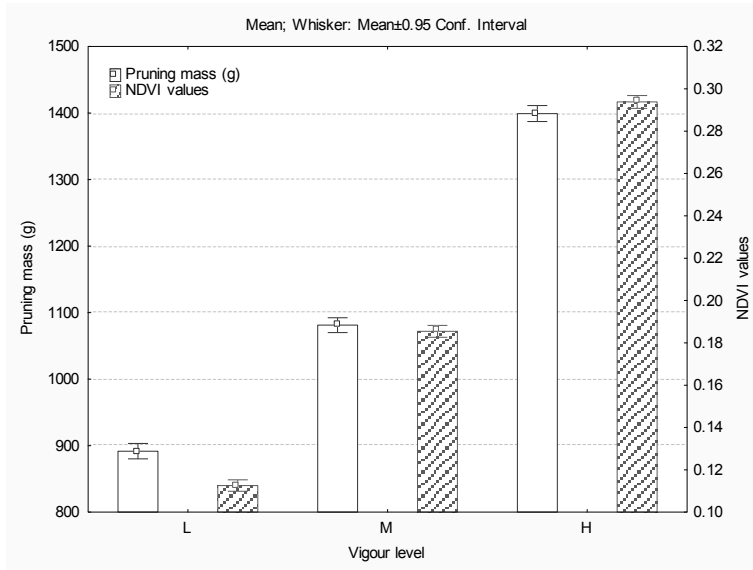

(a)

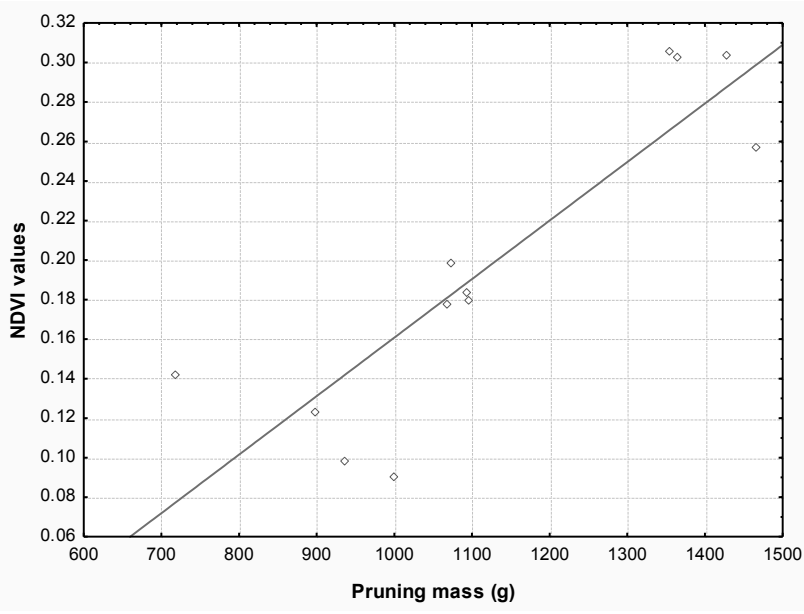

(b)

FIGURE 12

Means with error plot of pruning mass (g) per vine and NDVI values measured for plots manually subdivided into three distinct vigour levels (a). Scatter plot of NDVI values against pruning mass $(\mathrm{g})$ for sub-selected plots based on three vigour levels (b).

\section{CONCLUSIONS}

The objective of this research has been to develop a method of detecting vine rows in aerial imagery. This will enable more accurate determination of vine variability by focussing on the vine signal in the imagery, without including the influence of inter-row cover crop, shadows, soils, etc.

The analysis of the segmentation technique applied in this study, through rigorous ground-based verification, has shown results that appear to be reliable, although further testing is required. Both the vine row segmentation itself, as well as the vegetation index calculation, has improved previous classification methods, leading to improved estimators of vine biophysical measures.

The approach presented here differs from other techniques in that it can detect curved vine rows and is not limited to linear vine rows. This robust method of detecting vineyard rows makes use of both RGB and NIR images, whereas other methods typically only use the RGB image. The advantage of not using texture analysis is that it avoids the problem that neighbouring vineyard blocks with similar image texture are merged into a single vineyard block. As no region growing approach is used, there is no need for the identification of seed points, which potentially lends greater automation to the method presented here.

No hierarchal segmentation approach has been implemented in this study, but it is planned for future work. This will allow for the splitting of vine rows where the vine canopy has merged segments. Future work will investigate the correlation between vegetation indices and vine biophysical measures in more detail. The techniques developed in this study will also be applied to 
different vineyards in which growing conditions may vary, with the objective of quantifying the accuracies achieved for both image segmentation and the correlation between the vegetation index calculation and vine biophysical measures.

\section{LITERATURE CITED}

B\&H Photo Video, 2009. http://www.bhphotovideo.com (accessed 15 February 2009).

Bobillet, W., Da Costa, J., Germain, C., Lavialle, O. \& Grenier, G., 2003. Row detection in high resolution remote sensing images of vine fields. Precision agriculture: Papers from the 4th European Conference on Precision Agriculture, June 2003, Berlin, Germany.

Bramley, R., 2009. Progress in the development of precision viticulture - variation in yield, quality and soil properties in contrasting Australian vineyards. http:// www.crcv.com.au (accessed 15 February 2009).

Canon, 2009. http://www.usa.canon.com (accessed 15 February 2009).

Delenne, C., Rabatel, G., Agurto, V. \& Deshayes, M., 2006. Vine plot detection in aerial images using Fourier analysis. First International Conference on Objectbased Image Analysis (OBIA 2006), July 2006, Salzburg University, Austria

Franklin, S.E., Hall, R.J., Moskal, L.M., Maudie, A.J. \& Lavigne, M.B., 2000 Incorporating texture into classification of forest species composition from airborne multispectral images. Int. J. Remote Sensing 21, 61-79.

Hall, A., Louis, J. \& Lamb, D., 2003. Characterising and mapping vineyard canopy using high-spatial-resolution aerial multispectral images. Computers \& Geosciences 29(7), 813-822.

Johnson, L.F., Lobitz, B., Armstrong, R., Baldy, R., Weber, E., De Benedictis, J $\&$ Bosch, D.F., 1996. Airborne imaging aids vineyard canopy evaluation. Calif Agric. 50, 14-18.

Johnson L.F., Roczen, D.E., Youkhana, S.K., Nemani, R.R. \& Bosch, D.F., 2003. Mapping vineyard leaf area with multispectral satellite imagery. Comp. Electron. Agric. 38(1), 33-44
Lobitz, B., Johnson, L., Armstrong, R., Hlavka, C. \& Bell, C., 1997. Grapevine remote sensing analysis of phylloxera early stress (GRAPES): remote sensing analysis summary. NASA Technical Memorandum, No. 112218.

Maxmax, 2009. http://www.maxmax.com (accessed 15 February 2009).

Nikon, 2009. http://www.nikon-image.com (accessed 15 February 2009).

Photography Review, 2009. http://www.photographyreview.com/mfr/sigma/ 35mm-primes/PRD 84583 3111crx.aspx (accessed 15 February 2009).

Proffit, T., Bramley, R., Lamb, D. \& Winter, E., 2006. Precision viticulture - A new era in vineyard management and wine production. Winetitles, Australia. pp. $6-48$

Ranchin, T., Naert, B., Albuisson, M., Boyer, G. \& Astrand, P., 2001. An automatic method for vine detection in airborne imagery using wavelet transform and multiresolution analysis. Photogram. Engin. Remote Sensing 67, 91-98.

Rodríguez-Pérez, J., Álvarez-López, C., Miranda, D. \& Álvarez, M., 2008. Vineyard area estimation using medium spatial resolution satellite imagery. Spanish J. Agric. Res. 6(3), 441-452.

Rosin, P.L. \& Ioannidis, E., 2003. Evaluation of global image thresholding for change detection. Pattern Recognit. Lett. 24, 2345-2356.

Strever, A.E., 2003. A study of within-vineyard variability with conventional and remote sensing technology. MScAgric thesis. Stellenbosch University, Private Bag X1, 7602 Matieland (Stellenbosch), South Africa.

Tellaeche, A., BurgosArtizzu, X., Pajares, G., Ribeiro, A. \& Fernández-Quintanilla, C., 2008. A new vision-based approach to differential spraying in precision agriculture. Comp. Electron. Agric. 60, 144-155.

Tian, L. \& Slaughter, D., 1998. Environmentally adaptive segmentation algorithm for outdoor image segmentation. Comp. Electron. Agric. 21, 153-168.

Trudeau, R.J., 1993. Introduction to graph theory. Dover Publishers, New York.

Wassenaar, T., Robbes-Masson, J. \& Andrieux, P., 2002. Vineyard identification and description of spatial crop structure by per-field frequency analysis. Int. J. Remote Sensing 23(17), 3311-3325. 